\title{
Q1 Optical single sideband signal generation relying on a single-drive Mach-Zehnder modulator for radio over fibre communications
}

\author{
Varghese Antony Thomas ${ }^{凶}$, Mohammed El-Hajjar, Lajos Hanzo \\ School of Electronics and Computer Science, University of Southampton, Southampton, SO17 1BJ, UK \\ 凶-mail:vat1g10@ecs.soton.ac.uk
}

ISSN 1751-8628

Received on 26th May 2015

Revised on 11th October 2015

Accepted on 28th November 2015 doi: 10.1049/iet-com.2015.0498 www.ietdl.org

Abstract: Optical single sideband (OSSB) signals are conventionally generated using a dual-drive Mach-Zehnder modulator $(\mathrm{MZM})$ or by using a single-drive MZM in conjunction with a fibre Bragg grating filter. In this study, the authors propose and mathematically characterise a novel radio over fibre downlink in which an OSSB signal is generated using a single-drive, rather than dual-drive, MZM without any additional filter. Additionally, their proposed scheme can achieve optical upconversion of the electronic signal. Furthermore, in addition to cost advantages, the Q3 proposed scheme's BER performance is better than the classical OSSB signal generation scheme.

\section{Introduction}

Over the past decade, the number of wireless devices has witnessed 30 an exponential increase, besides becoming more bandwidth demanding [1]. The solution to the bandwidth crunch is a migration to higher radio-frequency $(\mathrm{RF})$ frequencies, where there is a large amount of unutilised spectrum [2]. However, the higher wireless attenuation experienced at higher frequencies demands the use of a cellular architecture relying on small cells. The use of small cells has the added advantage of the cellular system being able to support a larger number of users, because of a higher degree of frequency re-use. Fig. 1 shows a cellular system where the base station (BS) of each cell is connected to the wireless network's backhaul, which usually employs digital optical

40 communication. A full-fledged BS has several components having a high installation cost, especially when migrating to higher frequencies. Hence, the economics of setting up a cellular system in which each small cell is served by a BS is challenging, because of the large number of BSs needed.

Radio over fibre (ROF) is a promising solution to the challenge of having a large number of small cells with high-RF frequencies [3-6]. It is also possible for macrocells to co-exist with microcells, when relying on the ROF technique [7]. Fig. 1 also shows a cellular architecture relying on ROF, where multiple cells are served by a single BS by using radio access points (RAPs) that are connected

50 to the BS using optical fibre, which is often referred to as a distributed antenna system [1,8]. The RAP performs minimum signal processing and is not expensive, while most of the signal processing hardware is retained in the BS and is shared by multiple cells $[9,10]$. The advantages of the ROF-aided architecture include ability to implement multiple-input5 multiple-output techniques, ease of system upgrades, better resource allocation, better wireless coverage and higher power efficiency [1]. A comprehensive study of ROF techniques and its advantages has been provided in [1, 3, 9].

The 30 and $60 \mathrm{GHz}$ bands are viewed as potential frequency

60 bands capable of supporting high data rates in next-generation wireless networks relying on an ROF backhaul [2]. A challenge in the commercial exploitation of these high frequencies is the degrading nature of electronic components, especially of electronic mixers, at these high frequencies. The optical generation of the RF signals by employing optical upconversion is a potential solution to this challenge, which has been achieved in the proposed architecture by driving the Mach-Zehnder modulator (MZM) using a sawtooth signal.

The most commonly employed optical double sideband (ODSB) modulation suffers from fibre-dispersion-induced power attenuation, which becomes increasingly severe at higher RF carriers [1]. ODSB modulation may easily be implemented using a direct modulation of the laser source [6]. Optical single sideband (OSSB) modulation scheme was then proposed for overcoming this challenge [9]. Whilst being tolerant to the fibre-dispersion-induced power attenuation, the OSSB modulation scheme requires the employment of a dual-drive modulator [3]. Alternatively, one can employ a single-drive modulator, but will have to employ an extra temperature-sensitive fibre Bragg grating (FBG) optical filter for eliminating one of the sidebands [1]. In this paper, we propose a novel technique for generating an OSSB signal using a single-drive MZM without having to rely on an additional FBG filter, thereby providing substantial cost benefits.

Thus, the novel features of the proposed scheme are as follows:

- Generation of a dispersion-tolerant OSSB signal using a single-drive, rather than a dual-drive, MZM with no subsequent optical filtering, thereby resulting in significant reduction of the system cost.

- Optical upconversion of the OSSB signal using a sawtooth signal for avoiding the performance degradation imposed by high-RF mixers.

- Our solution outperforms the classical OSSB technique for similar link parameters.

This paper is organised as follows. The proposed system's architecture is discussed in Section 2, where a mathematical characterisation of the system is also provided in order to confirm its feasibility. Subsequently, in Section 3, we present the results of a simulation-based study that relies on realistic parameter values of commercially available optical equipments. Finally, our conclusions are presented in Section 4.

\section{Proposed system architecture and analysis}

In this section, we discuss how an OSSB signal can be generated using a single-drive MZM, where Fig. 2 shows the architecture of 

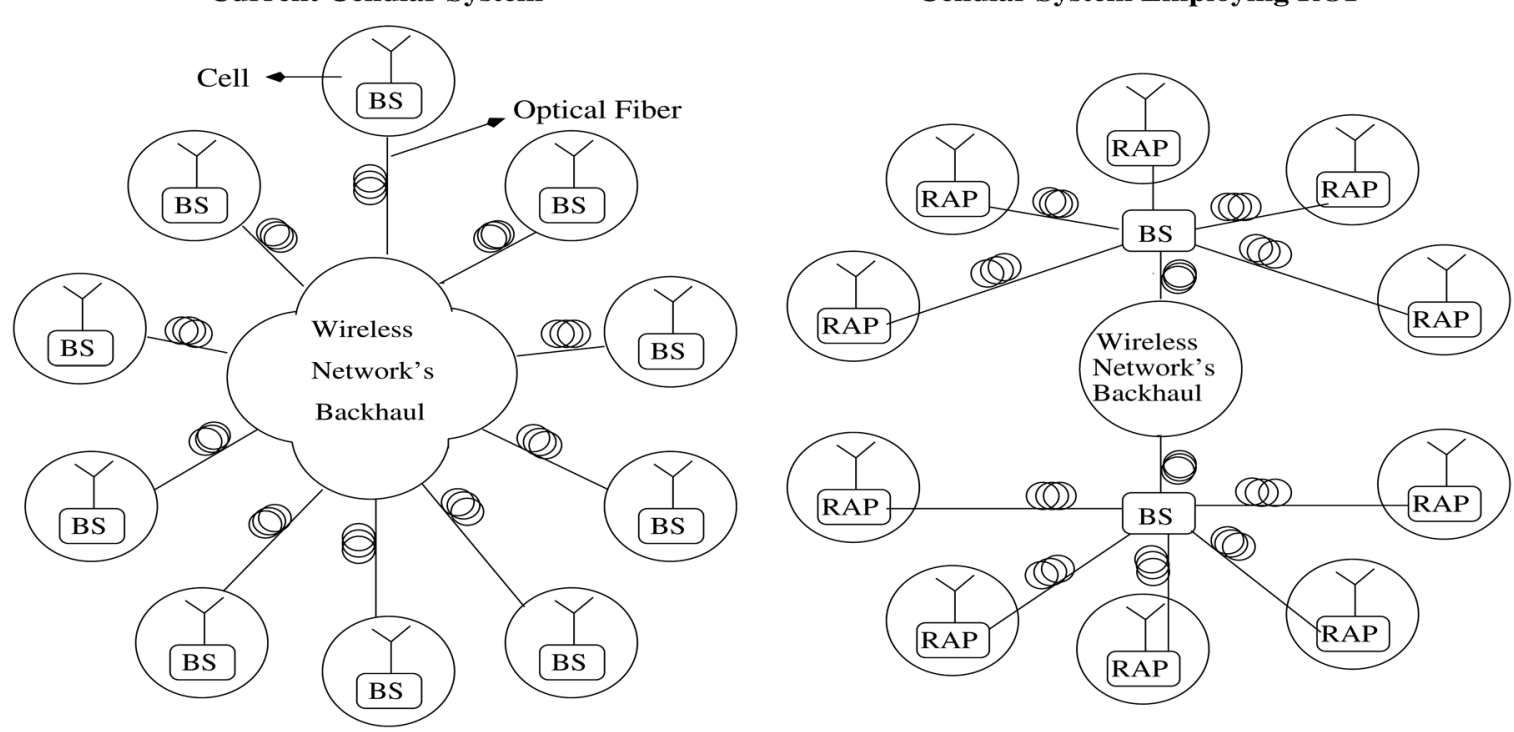

Fig. 1 Comparing the current cellular system to a cellular system employing ROF

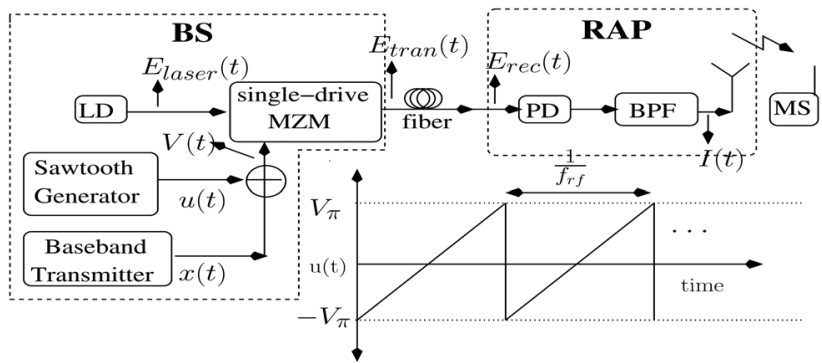

Fig. 2 Proposed system architecture relying on OSSB modulation

the proposed system, including the transmitter and receiver. First, we discuss the transmitter of the proposed architecture in Section 2.1, whereas the receiver of the proposed architecture is detailed subsequently in Section 2.2.

\subsection{Transmitter}

The laser diode (LD) in the BS of Fig. 2 operates at an optical frequency of $f_{c}$ hertz $(\mathrm{Hz})$ (wavelength $\lambda_{c}$ ) and at a power of $P_{\text {laser }}$ to generate an optical field of [1]

$$
E_{\text {laser }}(t)=\sqrt{2 P_{\text {laser }}} \mathrm{e}^{\mathrm{j} 2 \pi f_{c} t},
$$

where $E_{\text {laser }}(t)$ is fed to a single-drive MZM driven by $u(t)+x(t)$, with $x(t)$ being the baseband signal that is to be transmitted over the wireless link to the mobile station (MS), while $u(t)$ is the sawtooth signal of Fig. 2, formulated as

$$
u(t)=\operatorname{modulo}\left(2 f_{\mathrm{rf}} t V_{\pi}, 2 V_{\pi}\right)-V_{\pi},
$$

where $f_{\mathrm{rf}}$ is the RF carrier frequency, while $V_{\pi}$ is the switching voltage of the single-drive MZM. Note that $x(t)=+V$ for bit ' 1 ' phase-modulated symbols of $\mathrm{e}^{ \pm \mathrm{j}\left(V / V_{\pi}\right)}$ being received at the MS. Typically, phase-modulation employs phases of $(\pi / 2)$ and $-(\pi / 2)$ for bits ' 1 ' and ' 0 ', respectively. The optical output field of the

MZM having an insertion loss of $t_{\text {attn }}$ is

$$
\begin{aligned}
E_{\text {tran }}(t) & =\frac{\sqrt{t_{\text {attn }}}}{2}\left[1+\mathrm{e}^{\mathrm{j} \pi\left((x(t)+u(t)) / V_{\pi}\right)}\right] E_{\text {laser }}(t) \\
& =\frac{\sqrt{t_{\text {attn }}}}{2}\left[1-\mathrm{e}^{\mathrm{j}\left(\pi\left(x(t) / V_{\pi}\right)+2 \pi f_{\text {frt }} t\right)}\right] E_{\text {laser }}(t) .
\end{aligned}
$$

The spectrum of the OSSB signal $E_{\text {tran }}(t)$ is shown in Fig. 3. In Fig. 3, the optical carrier signal at $f_{\mathrm{c}} \mathrm{Hz}$ is represented by the first term in (3), whereas the optical sideband at $f_{\mathrm{c}}+f_{\mathrm{rf}} \mathrm{Hz}$ is represented by the second term in (3). Therefore, the system architecture of Fig. 2 is capable of generating an OSSB signal using a single-drive MZM. The generation of an ODSB signal using a sawtooth waveform applied to a dual-drive MZM was presented in [11].

Generating a sawtooth signal using an arbitrary waveform generator (AWG) would be an expensive proposition. However, the primary purpose of an AWG is to be able to generate arbitrary waveforms of many types, while our system needs only a sawtooth waveform generator. A more specific low-cost sawtooth signal generator can be readily constructed using only two op-amps, where the repetition frequency of the sawtooth waveform may be controlled using the resistance values employed $[12,13]$. [Many thanks to the anonymous reviewer for suggesting this

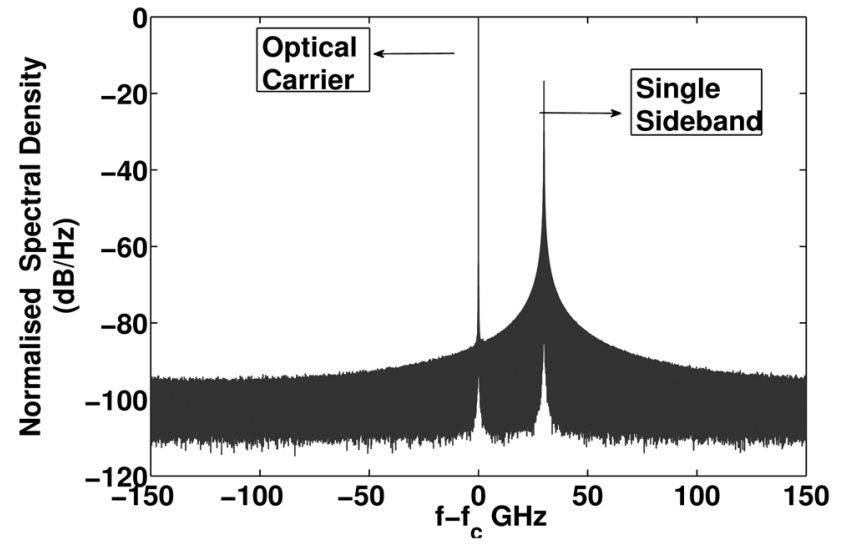

Fig. 3 Spectrum of the transmitted OSSB signal indicating a single sideband 
2 Q44 explanation.] In the next section, we discuss the receiver of the architecture in Fig. 2.

\subsection{Receiver}

270

The optical signal $E_{\text {tran }}(t)$ is transmitted through the optical fibre of length $L$ to the RAP. A phase shift $\phi_{\text {disp }}$ is induced by fibre-dispersion in the sideband with respect to the carrier, while additionally the signal is attenuated by the fibre [1]. The output of the photo-detector (PD) is then filtered using a band-pass filter (BPF) centred at $f_{\text {rf }} \mathrm{Hz}$ in order to separate the phase-modulated

275 RF component of (4). Thus, if $R$ is the responsivity of the photo-diode and BPF is the band-pass filtering function, then using (3), the photo-detected current is

280

$$
\begin{aligned}
I(t) & =\operatorname{BPF}\left\{R\left|E_{\mathrm{rec}}(t)\right|^{2}\right\} \\
& =\operatorname{BPF}\left\{R\left|\mathrm{e}^{(\alpha / 2) L} \frac{\sqrt{t_{\text {attn }}}}{2}\left[1-\mathrm{e}^{\mathrm{j}\left(\pi\left(x(t) / V_{\pi}\right)+2 \pi f_{\mathrm{rf}} t+\phi\right)}\right] E_{\text {laser }}(t)\right|^{2}\right\} \\
& =-\operatorname{Re}^{\alpha L} t_{\text {attn }} P_{\text {laser }} \cos \left(2 \pi f_{\mathrm{rf}} t+\pi \frac{x(t)}{V_{\pi}}+\phi\right),
\end{aligned}
$$

where $\alpha$ is the fibre attenuation parameter. Thus, it can be seen from (4) that the system architecture of Fig. 2 can achieve optical upconversion of the baseband signal $x(t)$ to the RF signal $I(t)$ using the sawtooth signal $u(t)$. If $x(t)$ is the two-level waveform

290 discussed in Section 2.1, then $I(t)$ would be a binary phase-shift keying modulated signal. The RF signal is then amplified and transmitted over the wireless link to the MS, where it is synchronously downconverted [14]. The RF signal is a phase-modulated upconverted version of the complex baseband

295 signal $\mathrm{e}^{\mathrm{j}\left(\pi\left(x(t) / V_{\pi}\right)+\phi_{\text {disp }}\right)}$. The fibre-dispersion rotates the symbols by a constant $\phi_{\text {disp. }}$. This can be considered as part of the wireless channel coefficient, when estimating the channel and performing phase rotation estimation. Hence, it is also readily estimated by the wireless channel estimation technique employed at the MS. In other words, if the wireless channel has a coefficient of $h$, then the channel coefficient obtained from the estimation technique becomes $h \mathrm{e}^{\mathrm{j} \phi}$.

Just like other modulation scenarios, a practical implementation of the proposed architecture may employ a non-ideal MZM. An $n$ th-order Bessel function may be used for representing a non-ideal

305 MZM's output [5]. Non-linear products such as intermodulation and harmonics are generated by these imperfections [5]. However, we hasten to add that, just like the classical OSSB, these non-linear signals may be significantly suppressed by optical filtering or/and by electronic filtering before and/or after photo-detection, respectively.

Now that we have discussed the mathematical feasibility of the proposed architecture, we move on to presenting the results that were obtained using a simulation-based study employing realistic system parameters.

315

\section{Simulation results and analysis}

Q5 The theoretical discussion in Section 2 did neither consider the effect of optical and electronic noise nor was the impact of the non-ideal nature of the optical components considered. In our simulations, we consider a realistic LD which has a non-zero linewidth and adds both phase noise as well as relative intensity noise (RIN). A non-ideal, realistic optical modulator having a finite extinction ratio as well as non-zero insertion loss is employed in these simulations. We have also analysed the effect of driving this 325 optical modulator using a non-ideal sawtooth signal. We have ensured that the optical power entering the MZM is not higher than $0 \mathrm{dBm}$. In fact, the highest optical power entering the MZM in our simulations was $-15 \mathrm{dBm}$. Additionally, this simulationbased study employs an optical fibre that imposes optical optical signal. Finally, we assume a PD that imposes thermal noise, shot noise as well as dark current on the signal. Table 1 provides the simulation parameters employed in our study [15]. The optical fibre propagation was simulated using the split step Fourier method [15].

We now show that the technique works well with realistic MZMs, unlike the MZM having an ideal extinction ratio $\epsilon$ of infinity assumed in (3). Commercial MZMs have

$$
E_{\text {tran }}(t)=\frac{\sqrt{t_{\text {attn }}}}{2}\left[1+\gamma \mathrm{e}^{\mathrm{j}\left(\pi V(t) / V_{\pi}\right)}\right] E_{\text {laser }}(t) .
$$

with $\gamma=(\sqrt{\epsilon}-1) /(\sqrt{\epsilon}+1)<1$. On reformulating (4), we arrive at

$$
I(t)=-\gamma R \mathrm{e}^{\alpha L} t_{\text {attn }} P_{\text {laser }} \cos \left(2 \pi f_{\mathrm{rf}} t+\pi \frac{x(t)}{V_{\pi}}+\phi\right) .
$$

Thus, a non-ideal extinction ratio weakens the photo-detected RF signal and results in a BER degradation, which is quantified in Fig. 4 for various extinction ratios. As seen in Fig. 4, realistic MZMs having extinction ratios of about 20-30 dB impose a tolerable BER degradation compared with an ideal MZM with $\epsilon=\infty$ decibels $(\mathrm{dB})$. An ideal MZM has an extinction ratio of $\infty \mathrm{dB}$. In the simulation of Fig. 4, we operated the ROF link at an optical power that would yield a BER of $\sim 10^{-9}$ for an ideal MZM, where this BER was due to the noise imposed by the ROF link. However, an additional BER degradation is imposed, if the MZM has a non-ideal extinction ratio, as seen from Fig. 4. Hence, the

\begin{tabular}{|c|c|c|c|}
\hline Parameter & Value & Parameter & Value \\
\hline bit rate of $x(t)$ & $32 \mathrm{Mbps}$ & $\begin{array}{c}\text { MZM insertion loss } \\
t_{\text {attn }}\end{array}$ & $4 \mathrm{~dB}$ \\
\hline laser $\mathrm{RIN}, \mathrm{dBc} / \mathrm{Hz}$ & -155 & MZM extinction ratio $\epsilon$ & $30 \mathrm{~dB}$ \\
\hline optical wavelength $\lambda_{\mathrm{c}}$ & $1550 \mathrm{~nm}$ & fibre length $L$ & $20 \mathrm{~km}$ \\
\hline MZM switching & $6 \mathrm{~V}$ & PD trans-impedance & $125 \Omega$ \\
\hline voltage $V_{\pi}$ & & laser linewidth & $10 \mathrm{MHz}$ \\
\hline fibre dispersion $D$ & $16 \mathrm{ps} / \mathrm{km} \mathrm{nm}$ & $\begin{array}{l}\text { fibre non-linearity } \\
\text { dark current }\end{array}$ & $\begin{array}{c}1.2 \mathrm{~W} / \mathrm{km} \\
1 \mathrm{nA}\end{array}$ \\
\hline fibre attenuation $\alpha$ & $0.2 \mathrm{~dB} / \mathrm{km}$ & photo-receiver NF & $6 \mathrm{~dB}$ \\
\hline PD responsivity $R$ & $0.8 \mathrm{~A} / \mathrm{W}$ & $f_{\text {rf }}$ & $30 \mathrm{GHz}$ \\
\hline
\end{tabular}
BER floor in Fig. 4 indicates that the BER performance would approach its value for the case of an ideal MZM. Hence, we employ an extinction ratio of $30 \mathrm{~dB}$ in all our subsequent simulations.

Table 1 Parameter values employed in the ROF link

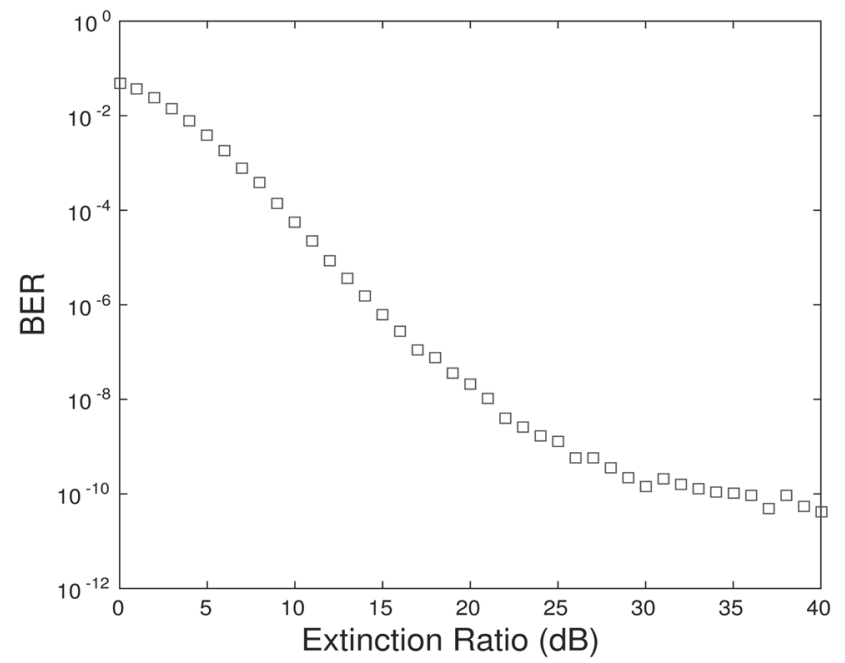

Fig. 4 System performance for non-ideal MZM having a finite extinction ratio 
The discussion in Section 2 assumed an ideal sawtooth signal capable of instantaneously switching from $V_{\pi}$ to $-V_{\pi}$ volts. However, realistic realisations have a non-zero fall time. Fig. 5 shows a sawtooth signal with a non-zero fall time of $T_{\text {fall }}$ seconds, where the repetition period of the sawtooth signal is $T_{\text {period }}$ seconds. Fig. 6 shows the BER degradations for various non-zero fall times $T_{\text {fall }}$ as a percentage of $T_{\text {period }}$, i.e. $\left(T_{\text {fall }} / T_{\text {period }}\right) \times 100$, where the laser was operated at a transmit power that would result in a BER of $\sim 10^{-5}$ for a near-ideal sawtooth signal. It can be seen from Fig. 6 that a fall time of up to $10 \%$ of the repetition period of the sawtooth signal results in a tolerable BER degradation, if we allow a BER of $2 \times 10^{-4}$. Hence, in the following paragraphs we employ a fall time of $10 \%$ of the repetition period of the sawtooth signal.

The generation of the classical OSSB signal as well as of the chirp-free ODSB signal are discussed in [1]. Fibre dispersion results in the photo-detected power being attenuated for the case of the chirp-free ODSB signal according to [1]

$$
P_{\mathrm{rf}} \propto \cos ^{2}\left[\frac{\pi \cdot L \cdot D \cdot \lambda_{\mathrm{c}}^{2} \cdot f_{\mathrm{rf}}^{2}}{c}\right],
$$

where $c$ is the speed of light in vacuum, which results in a BER degradation. The normalised RF power transfer in the photo-detected RF signal for the case of a dispersive fibre is shown in Fig. 7, where the normalisation was carried out with respect to the photo-detected RF power for the case of no fibre dispersion. The photo-detected RF signal does not suffer from dispersion-induced power attenuation, when employing the

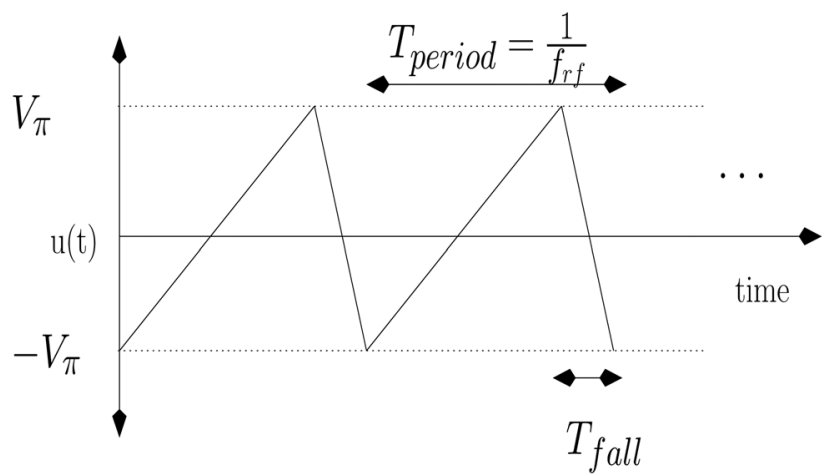

Fig. 5 Non-ideal sawtooth signal with a non-zero fall time

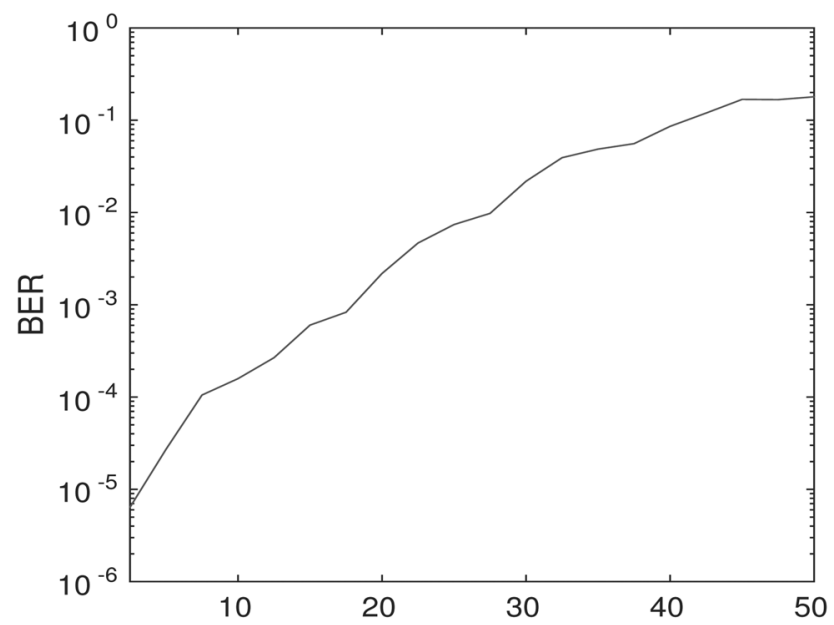

Fall time ( $\%$ of sawtooth signal's repetition period)

Fig. 6 System performance for non-ideal sawtooth signal having a non-zero fall time

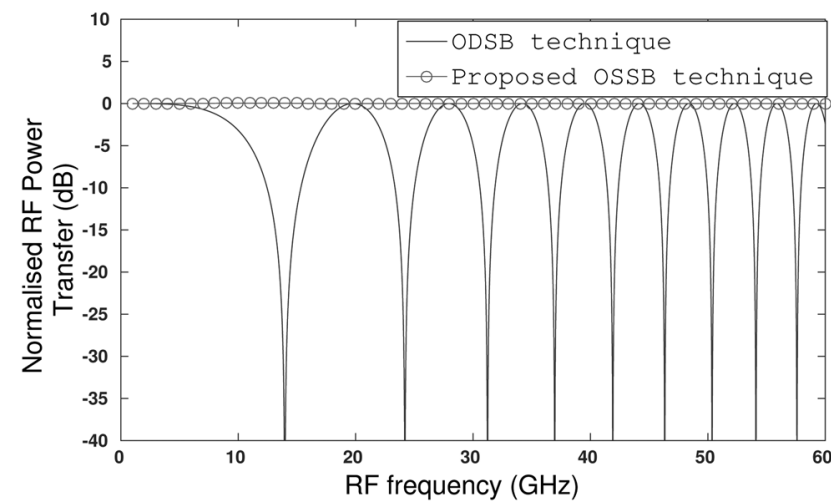

465

Fig. 7 RF power transfer of the fibre link normalised with respect to that in a zero-dispersion scenario

classical OSSB modulation that relies on a dual-drive MZM [3]. It can be seen from Fig. 7 that our new OSSB modulation technique also provides the same advantage.

In the proposed architecture, the single sideband is generated by using the phase-shifter in the arm of a single-drive MZM for frequency shifting the optical sideband carrying a baseband electronic signal. Hence, as seen from Fig. 3, the proposed technique does not generate harmonics. On the other hand, Fig. 8 shows the optical spectrum of the classical OSSB signal, where we can observe the generation of harmonics because intensity-, rather than phase-modulation is employed. Fig. 9 shows the non-linear intensity transmittance of a dual-drive MZM, where the classical OSSB signal is generated by employing a bias voltage of $V_{\pi} / 2$. The BER attained using the classical OSSB modulation depends on the maximum drive voltage employed. Explicitly, employing a higher drive voltage generates a stronger RF signal, but it also generates stronger harmonics. It can be seen from Fig. 9 that the drive voltage has an absolute maximum of $V_{\pi}=6 \mathrm{~V}$ peak-to-peak. Drive voltages higher than $V_{\pi}=6 \mathrm{~V}$ peak-to-peak would result in multiple values of the RF signal being transmitted using the same optical power, i.e. the faithful one-to-one mapping between the RF voltage and optical power is lost. However, operating at a value close to the absolute maximum of $6 \mathrm{~V}$ peak-to-peak generates strong non-linear signals. The suppression of harmonics becomes important, when employing multiple RF signals, because the harmonics of one RF signal may corrupt another RF signal. Additionally, a higher drive voltage also results in stronger intermodulation products, when employing multiple RF signals, where the third-order intermodulation products lie very close to the RF signals transmitted. Figs. 10 and 11 show the suppression

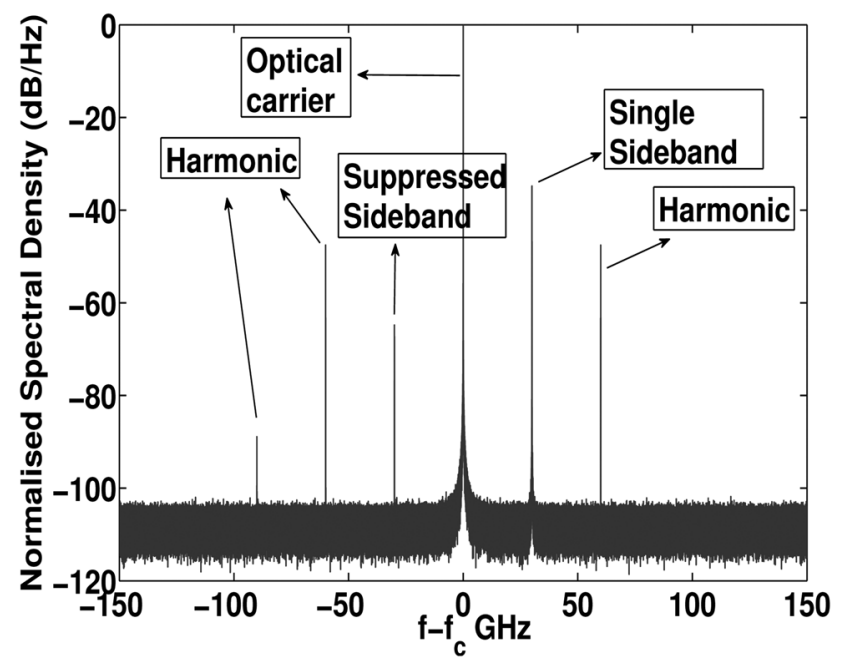

Fig. 8 Optical spectrum of the classical OSSB signal 
Fig. $11 R F$ signal to harmonic power ratio for various values of the maximum drive voltage

of the optical and photo-detected harmonics, respectively, with respect to the desired signal for various values of the maximum drive voltage. It can be seen from Figs. 10 and 11 that a

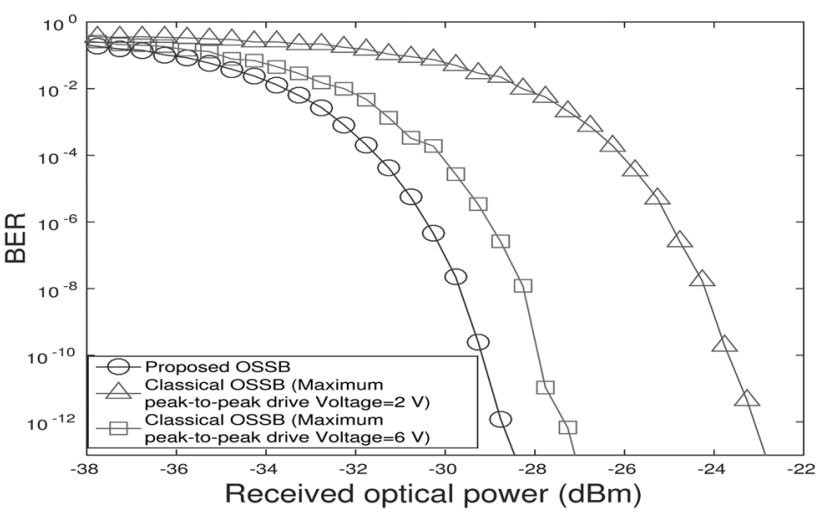

Fig. $12 B E R$ performance of the proposed scheme to that of the classical OSSB scheme

differential maximum RF voltage of $2 \mathrm{~V}$ peak-to-peak results in harmonics, which can be effectively suppressed by practical electronic filters. Nevertheless, in our simulations we consider both scenarios, i.e. scenarios having a maximum drive voltage of $2 \mathrm{~V}$ peak-to-peak and $6 \mathrm{~V}$ peak-to-peak. Note that the amplitude of the drive voltage is typically half of its peak-to-peak value.

Fig. 12 shows the BER performance of the optical link proposed in this paper for the simulation parameters of Table 1. Fig. 12 shows the performance attained by the link in Fig. 2, when employing the classical dual-drive MZM-based OSSB signal generation, instead of the proposed single-drive MZM. A maximum differential RF voltage of $2 \mathrm{~V}$ peak-to-peak and $6 \mathrm{~V}$ peak-to-peak was applied to the two arms of the dual-drive MZM. It can be seen from Fig. 12 that the performance of the proposed technique is better than that of the classical OSSB technique. Explicitly, the BER performance of the proposed scheme is better than the best-case performance achieved by the classical OSSB scheme using a maximum differential RF voltage of $6 \mathrm{~V}$ peak-to-peak. On the other hand, the cost of these performance improvements is the constraint of transmit optical modulation using a baseband electronic signal, which makes our proposed scheme suitable for only downlink transmission. Additionally, there is the constraint of having to employ phase modulation of RF signals, as seen in (4).

The classical approach of generating an OSSB signal relies on a dual-drive MZM [1]. A single-drive MZM has only a single active arm with its associated driving circuitry, whereas the dual-drive MZM consists of two active arms along with the associated pair of electronic circuitry. Hence, the cost of a dual-drive MZM is higher than that of a single-drive MZM. The second classical approach of generating an OSSB using a single-drive MZM along with an FBG filter suffers from an increased cost due to the need for optical filtering [1]. Indeed, both the single- and dual-drive MZMs are commercially available. However, the difference in the cost of OSSB generation between relying on the proposed technique using only a single-drive MZM and on the classical methods, certainly becomes significant, when viewed in conjunction with the fact that over three million small cells are forecast for 2016 [16]. Moreover, this number is expected to rapidly increase in the subsequent years. Any difference in the cost of generation would become significant, when considering the large number of optical transmitters that would be needed.

\section{Conclusions}

An OSSB modulation scheme was designed that relies on a single-drive, rather than dual-drive, MZM, while dispensing with the employment of an optical filter. Additionally, optical upconversion of the baseband signal to RF was facilitated by driving the single-drive MZM using a sawtooth signal. The proposed OSSB scheme was shown to outperform the conventional dual-drive MZM-based OSSB technique. 


\section{Acknowledgments}

Q3 The financial support of the EU's Concerto Project, as well as that of the EPSRC, UK under the auspices of the India-UK Advanced 665 Technology Centre and that of the European Research Council under its Advanced Fellow Grant is gratefully acknowledged.

\section{References}

1 Thomas, V., El-Hajjar, M., Hanzo, L.: 'Performance improvement and cost reduction techniques for radio over fiber communications', IEEE Commun. Surv. Tutor., 2015, 17, pp. 627-670

2 Rappaport, T., Sun, S., Mayzus, R., et al: 'Millimeter wave mobile communications for 5G cellular: it will work!', IEEE Access, 2013, 1, pp. 335-349

3 Thomas, V., El-Hajjar, M., Hanzo, L.: 'Millimeter-wave radio over fiber optical upconversion techniques relying on link non-linearity', IEEE Commun. Surv. Tutor., 2015, PP, early access

4 Fernando, X., Sesay, A. 'Adaptive asymmetric linearization of radio over fiber links for wireless access', IEEE Trans. Veh. Technol., 2002, 51, pp. 1576-1586

5 Fernando, X.N.: 'Radio over fiber for wireless communications: from fundamentals to advanced topics' (Wiley-IEEE, Chichester, UK, 2014, 1st edn.)
6 Zhang, Y., Li, J., Chen, H., et al.: 'Clip-and-filter-based crest factor reduction and digital predistortion for WLAN-over-fiber links', IEEE Photonics Technol. Lett. 2014, 26, pp. 2315-2318

7 Al-Dulaimi, A., Al-Raweshidy, H., Cosmas, J., et al.: 'Cognitive mesh networks', IEEE Veh. Technol. Mag., 2010, 5, pp. 54-60

8 Al-Raweshidy, H., Komaki, S.: 'Radio over fiber technologies for mobile communications networks' (Artech House Inc., Norwood, MA, USA, 2002, 1st edn.)

9 Beas, J., Castanon, G., Aldaya, I., et al.: 'Millimeter-wave frequency radio over fiber systems: a survey', IEEE Commun. Surv. Tutor., 2013, 15, pp. 1593-1619

10 Koshy, B., Shankar, P.: 'Spread-spectrum techniques for fiber-fed microcellular networks', IEEE Trans. Veh. Technol., 1999, 48, pp. 847-857

11 Thomas, V., El-Hajjar, M., Hanzo, L.: 'Single MZM-based, fiber-dispersiontolerant ODSB radio-over-fiber signalling improves the wireless downlink by enabling STBC transmission from each radio access point', IEEE Commun Lett., 2015, early access

12 Salivahanan, S., Kanchana Bhaaskaran, V.S.: 'Linear integrated circuits' (Tata McGraw-Hill, New Delhi, India, 2008, 1st edn.)

13 Bakshi, U.A., Godse, A.P.: 'Linear IC applications' (Technical Publications, Pune, India, 2010, 1st edn.)

14 Goldsmith, A.: 'Wireless communications' (Cambridge University Press, NY, USA, 2005, 1st edn.

15 Agrawal, G.P.: 'Fiber-optic communication systems' (John Wiley and Sons, 2002, 3rd edn.)

16 'Small cell forum'. Available at http://www.smallcellforum.org 


\section{COM20150498}

795 Author Queries

Varghese Antony Thomas, Mohammed El-Hajjar, Lajos Hanzo

Q1 Please confirm the changes made in the article title as 'Optical single sideband signal generation relying on a single-drive MachZehnder modulator for radio over fibre communications'. Please note that it is the IET's house style to remove words such as 'Novel', 'New' and 'Study of' as well as 'A', 'An' and 'The'.

Q2 Please check and confirm the corresponding author email id.

Q3 Please provide expansion for the abbreviations BER, EU, EPSRC, NF.

Q4 Footnote has been moved to text as required by journal style. Please check and confirm that it has been located correctly within the text.

Please noise nor was the impact of the non-ideal nature of the optical components considered'.

Q6 Please update Refs. [3, 11] with volume number and page range.

Q7 Please provide the publisher location in Ref. [15] 